Nouvelles perspectives en sciences sociales

Revue internationale de systémique complexe et d'études relationnelles

\title{
Économie et société. Pistes de sortie de crise, Louis Favreau et Ernesto Molina, Presses de l'Université du Québec, coll. " Initiatives », 2011
}

\section{Mircea Vultur}

Volume 8, numéro 2, mai 2013

URI : https://id.erudit.org/iderudit/1016476ar

DOI : https://doi.org/10.7202/1016476ar

Aller au sommaire du numéro

\section{Éditeur(s)}

Prise de parole

\section{ISSN}

1712-8307 (imprimé)

1918-7475 (numérique)

Découvrir la revue

Citer ce compte rendu

Vultur, M. (2013). Compte rendu de [Économie et société. Pistes de sortie de crise, Louis Favreau et Ernesto Molina, Presses de l'Université du Québec, coll. "Initiatives ", 2011]. Nouvelles perspectives en sciences sociales, 8(2), 209-214. https://doi.org/10.7202/1016476ar d'utilisation que vous pouvez consulter en ligne. 


\section{Comptes rendus de lecture}

\section{Économie et société. Pistes de sortie de crise}

Louis Favreau et Ernesto Molina, Presses de l'Université du Québec, coll. «Initiatives », 2011.

\section{PAR Mircea Vultur \\ Centre Urbanisation Culture Société, Québec}

ublié à la suite d'une grande conférence internationale sur
le mouvement coopératif qui s'est tenue à Lévis, au Québec, en septembre 2010, le livre de Louis Favreau et Ernesto Molina examine les rapports contemporains entre économie et société, marqués par la crise économique de 2008, et se propose d'offrir des pistes de solution pour la sortie de cette crise. Leur questionnement général, qui reprend celui des organisateurs de la Conférence de Lévis, est énoncé de la manière suivante : "Comment réinventer l'économie et l'orienter vers un type de société qui entend respecter les équilibres écologiques et en même temps être porteuse de justice économique et sociale? " (p. 5). Pour y répondre, les auteurs entreprennent une critique du mode d'organisation de l'économie capitaliste et mettent en perspective les valences positives de l'économie solidaire et du mouvement coopératif en général (chapitre 1) et dans plusieurs domaines de l'action sociale tels l'organisation de la production (chapitre 2), l'environnement (chapitre 2), le mode d'intervention de l'État (chapitre 4), l'agriculture et les territoires (chapitre 5), les rapports Nord-Sud (chapitre 6), les mouvements sociaux (chapitre 
7). Dans le dernier chapitre du livre, les auteurs en appellent à un " projet de société du mouvement coopératif » en 6 chantiers et 24 propositions qui relèvent cependant moins d'une évaluation réaliste des évolutions sociales et économiques actuelles que d'un acte de foi.

Bons connaisseurs des modèles alternatifs de développement et des recherches sur le mouvement collectif (étant eux-mêmes des chercheurs spécialisés dans ce domaine), les auteurs nous présentent des informations intéressantes sur l'histoire de l'économie solidaire, sur les particularités de ses structures et son mode de fonctionnement, notamment dans le cas du Québec. Ils examinent ses métamorphoses et ses reconfigurations au cours des vingt dernières années, période caractérisée par une série de changements : "explosion des pratiques coopératives un peu partout dans le monde; transformation des grandes organisation sociales nées ou reconstituées dans l'après-guerre (mouvement syndical, mouvement des agriculteurs, mouvement coopératif); émergence de nouveaux réseaux ou de nouvelles organisations (mouvement de la consommation responsable, commerce équitable, réseaux de développement local) »(p. 11). Selon les auteurs, ce contexte fait de l'économie solidaire et des entreprises à propriété collective, des éléments susceptibles d'offrir une réponse appropriée à la crise globale du capitalisme qui a cours actuellement. Cette crise, non seulement "financière et économique " mais aussi " écologique et sociale " représente une occasion de mobiliser la " pensée économique alternative » et de " reconfigurer l'ensemble de l'économie et de la société » (p. 12). Selon Favreau et Molina, la crise " que nous venons de vivre en 2008 n'est pas une petite crise cyclique mais une grande crise comparable avec celle des années 1930 par son ampleur et sa profondeur ", mettant en relief " la cruauté d'un système qui imposait la logique du plus grand profit» (p. 23). L'économie solidaire devient, dans ce contexte, une solution viable au capitalisme, système somme toute, coupable des problèmes socioéconomiques actuels et susceptible d'engendrer des destructions planétaires irrémédiables. Sur ce plan et sans contredire totale- 
ment les analyses des auteurs, il faut néanmoins mettre un bémol sur cette vision catastrophique de notre monde actuel et de son évolution future de même que sur la nature de ce bouc émissaire qu'est le capitalisme. Pleins de bonne foi et soucieux de l'avenir de l'humanité, les auteurs ignorent cependant l'histoire des évolutions sociales du monde occidental qui montre que le capitalisme a su surmonter ses crises, qu'il a eu une extraordinaire force de "se sauver" par des transformations internes qui ont préservé " la logique de la recherche du profit " et l'intérêt individuel comme force motrice de l'action collective. Soulignons également que, dans leur comparaison des crises de 1930 et de 2008, les auteurs ne tiennent pas compte d'une série d'éléments de contexte qui font en sorte que les deux phénomènes sont différents et que le " capitalisme " contemporain est mieux armé que celui des années 30 pour faire face aux éléments destructeurs de la crise.

Dans cette perspective, pour proposer des pistes de sortie de crise (thème au centre de leur questionnement), les auteurs auraient dû analyser les causes qui ont provoqué les bouleversements de 2008, situer leurs critiques dans le système capitaliste et non pas faire, de manière rhétorique, le procès du marché comme régulateur. La crise de 2008 a inspiré une abondante littérature qui montre la complexité de ses causes. Ces causes sont liées non seulement au fonctionnement du marché mais aussi aux erreurs répétées de politique économique et monétaire, à l'absence de morale des responsables politiques, au soutien quasi systématique des États aux industries en déclin et plus profondément, au raccourcissement de l'horizon temporel de nos processus décisionnels ${ }^{1}$. Proposer des pistes de sortie de crise sans analyser plus en détail les causes de ce phénomène me semble prendre un raccourci intellectuel d'assez forte envergure.

Par ailleurs, l'idée que l'économie solidaire est une alternative à la crise actuelle du capitalisme est, sans doute, à prendre en compte, mais il me semble difficile de faire la promotion de ce

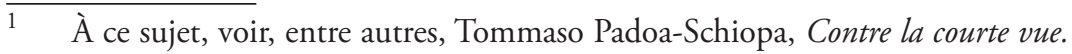
Entretiens sur le Grand Krach, Paris, Odile Jacob, 2009. 
type d'économie dans le contexte actuel d'individualisation croissante des relations de travail, d'un " capitalisme de la singularité » qui affirme de plus en plus la réalisation individuelle au travail. Les salariés ne sont plus équivalents, le mérite personnel s'impose de plus en plus comme valeur et référence d'action au travail $^{2}$. Si Favreau et Molina montrent dans leur livre le développement important de l'économie sociale au cours des dernières années, au Québec et sur le plan international, il faut aussi constater que cette économie affirme sa vitalité au moment où elle dépérit comme forme de production adaptée à l'ethos du travail contemporain. Le mouvement coopératif semble progresser en même temps que régresse la morale de l'égalité.

Outre la promotion des vertus de l'économie solidaire, les auteurs se font les défenseurs de "l'état social aux prises avec l'offensive néolibérale » et les promoteurs des entreprises collectives et du " renouveau collectif " sans toutefois offrir des idées claires en ce sens. L'État apparaît comme nécessaire aux clientèles variables dépendantes de son action d'expropriation et de redistribution, et le " renouveau » se trouve beaucoup dans la rhétorique. L'esprit du livre est traversé aussi de l'idée favorable à l'altermondialisation voire à la démondialisation, parce que, selon les auteurs, l'actuelle "mondialisation néolibérale " accouche d'un monde mauvais dont le potentiel de progrès induit des phénomènes négatifs tels les inégalités Nord-Sud (concept qui aujourd'hui n'a plus vraiment de signification). Certes, la mondialisation est une forme de modernité qui a ses côtés sombres, mais l'idée qu'elle a accentué fortement les inégalités entre les pays du Nord et les pays du Sud doit être relativisée parce que, comme les données statistiques et les recherches récentes le démontrent, entre 1990 et aujourd'hui, période de forte accélération de la mondialisation, la croissance du revenu moyen dans les pays avancés a été beaucoup moins rapide que dans les pays émergents, et que c'est à l'intérieur de certaines pays développés

$\overline{2}$ Daniel Mercure et Mircea Vultur, La Signification du travail. Nouveau modèle productif et ethos du travail au Québec, Québec, Presses de l'Université Laval, 2010. 
(dont les États-Unis et le Canada) ou en Chine que les inégalités au sein de la population ont augmenté. En fait, dans l'ensemble, on assiste à une baisse significative de l'inégalité entre tous les citoyens du monde ${ }^{3}$.

De manière générale, le fil conducteur qui traverse ce livre est une critique marxiste du capitalisme et de la mondialisation " néoliberale " axée sur deux postulats fondamentaux sous-jacents aux analyses. Le premier soutient que la ligne de fracture d'une société passe par l'opposition entre les possesseurs du capital (ou les entreprises capitalistes) et la classe ouvrière salariée. Les premiers exploitent les travailleurs en accaparant la richesse produite par leur travail. Le deuxième postulat qui imprègne les critiques produites dans ce livre pose que le capitalisme peut être dépassé sur son propre terrain, qu'il est possible de le remplacer par un système plus juste et plus équitable et que l'économie solidaire est une composante de ce système. Le livre nous suggère qu'un autre monde, susceptible d'éradiquer les maux produits par le capitalisme, est possible. Une morale collectiviste et anti-individualiste traverse les réflexions des auteurs qui font écho à une tendance qui s'affirme d'ailleurs aujourd'hui de plus en plus dans les sociétés occidentales comme conséquence de l'effondrement de protections offertes par le système fordiste.

Même si le livre cautionne une critique stérile du capitalisme et de la mondialisation, il apporte des éléments d'information pertinents sur l'économie solidaire et ses différentes composantes, sur son évolution et son état de développement dans le monde d'aujourd'hui, susceptibles d'intéresser diverses catégories de lecteurs. Les éclairages que le livre de Favreau et Molina propose nous permettent d'envisager plus clairement la question de l'économie solidaire qui, défendue fortement pas ses partisans, n'est pas encore près d'être refoulée. En accord avec l'esprit de leurs réflexions, je crois que nous avons besoin d'idées alternatives et de la promotion de valeurs qui soient autres que celles d'un marché planétaire unifié par la consommation et la circulation

3 Voir à ce sujet, Christian Morrisson et Fabrice Murtin, «Inégalités et pauvreté depuis trois siècles ", Commentaire, no 138, 2012, p. 305-319. 
des marchandises. Je doute cependant que l'économie solidaire soit une alternative viable au capitalisme et surtout à la sortie de la crise actuelle. Je crois que ce type d'économie comporte des éléments positifs à l'intérieur du système de marché, qui reste jusqu'à cette date le plus efficace pour la promotion du développement économique. On peut certes reconnaître le caractère discutable de la justesse des valeurs et des critères du capitalisme libéral sans s'insurger contre la référence au marché et fuir dans la recherche d'un alter-système. Si le livre de Favreau et Molina nous oriente vers cet alter-système, il ne démontre pas qu'il fait partie des alternatives économiques viables à la crise de 2008 . Comme je l'ai dit en début de ce compte rendu, leur " projet de société » relève plus d'un acte de foi que d'une évolution réaliste de l'économie. L'utopie collectiviste a, certes, toujours ses partisans mais, dans la société contemporaine, la culture individualiste est plus forte que jamais. Avec ou sans économie solidaire, l'individualisme démocratique laissé à lui-même peut produire une cohésion sociale raisonnable. Et nous pouvons retrouver notre capacité d'action collective sans tomber dans l'idéologie de l'« ennemi à abattre » et dans l'illusion révolutionnaire. 